\title{
Tachometer Device
}

National Cancer Institute

\section{Source}

National Cancer Institute. Tachometer Device. NCI Thesaurus. Code C50060.

A device designed to measure a speed of rotation. 